\title{
Transformation of Long Waves in a Canal of Variable Section
}

\author{
Kazimierz Szmidt, Benedykt Hedzielski \\ Institute of Hydro-Engineering, Polish Academy of Sciences, ul. Kościerska 7, 80-328 Gdańsk, Poland, \\ e-mails: jks@ibwpan.gda.pl, ben@ibwpan.gda.pl
}

(Received December 14, 2015; revised June 11, 2016)

\begin{abstract}
The paper deals with long water waves propagating in a straight canal of constant depth and variable section. In the formulation of this problem, a simplified, one-dimensional model is considered that is based on the assumption of a "columnar" fluid motion. To this end, a system of material coordinates is employed as independent variables in the description of this phenomenon. The main attention is focused on transient solutions corresponding to a fluid motion starting from rest. With respect to the initial value problem considered, we confine our attention to a finite domain fluid motion induced by a piston-type generator placed at the beginning of the canal. For a finite elapse of time, measured from the starting point, the solution in the finite fluid area mimics a solution within an infinite domain, inherent for wave propagation problems. The main goal of our investigations is to describe the evolution of the free surface (the wave height) at the smallest section of the canal. Numerical examples are provided to illustrate the model formulation developed in this paper. The accuracy of this approximate description is assessed by comparing its results with data obtained in hydraulic experiments performed in a laboratory flume.
\end{abstract}

Key words: long water wave, canal of variable section, enforcement of wave height, material variables

\section{Introduction}

Sea waves approaching coastal zones are potential sources of mechanical energy that can be converted into electric current. The density of the energy flux carried by water waves is much higher than that of wind energy, but its utilization is a difficult task. It strongly depends on natural local conditions associated with the variation in the sea level within the day and night periods. For instance, tidal ocean waves carry more energy than wind sea waves generated locally over relatively small areas. An example of the latter is the Baltic sea and, in particular, the Polish coastal zone. In order to absorb the mechanical wave energy and convert it into electric power, special devices are constructed dependent on local sea conditions. Among others, the simplest are generators based on the oscillating water column, which harness the water wave energy associated with an oscillating, floating body. Their effectiveness depends on the 
height of water waves, mainly standing waves, which force the body to move up and down with respect to the mean water level. As far as the Polish coast is considered, the heights of sea waves are relatively small, and therefore, in order to increase the utilization of wave energy, it would be necessary to construct a structure capable of amplifying the body oscillation, and thus the available wave energy. It seems reasonable to expect that a simple structure in the form of a canal with vertical sides gradually converging along with propagating waves, could collect the energy of waves arriving from a wider area, and thereby improve wave energy utilization. Accordingly, in order to assess the effectiveness of such a structure, a theoretical study on the transformation of water waves in a canal of variable section is carried out. In the theoretical investigation, we resort to an approximate modeling of the original task that can describe the main features of this phenomenon. In order to simplify our further discussion, we confine our attention to long waves. In a description of these waves, it is justified to employ the assumption of a "columnar motion," according to which horizontal displacements of fluid particles forming a vertical water column are the same during the entire fluid motion. This assumption makes it possible to reduce the number of independent parameters of the problem by one. In a formal way, it is also possible to reduce the problem description to a simpler, one-dimensional model in which all parameters of the fluid motion are expressed in terms of one space coordinate and time.

With respect to the above, the main focus of this research will be the investigation of the free-surface elevation changes associated with the variation in canal section along with the direction of water waves arriving from the open sea. Since two dimensions of the fluid domain, i.e. its depth and width, are much smaller than the fluid length, an approximate formulation is considered in which all parameters of the fluid motion are expressed in terms of one space coordinate and time. Such an approximation was employed by Lamb (1975) in a description of wave motion in a canal of variable section. In that formulation, based on the fundamental assumption of a hydrostatic pressure, a wave-like equation was derived that describes the evolution of the free surface of the fluid in time. In the case of a constant cross section of the canal, that equation reduces to a commonly used, well-known wave equation describing the propagation of small long water waves in fluid of constant depth. This equation corresponds to the space (Eulerian) system of coordinates. An analytic solution to a similar problem of a steady harmonic motion of the free surface of fluid in a canal of variable section (with vertical sides converging at a point) is presented in a monograph by McLachlan (1964). The solution is expressed in the form of a linear combination of the smallest order Bessel functions of the first and second kinds. It should be stressed, however, that this solution describes standing cylindrical waves, which may only be considered as an approximation of plane waves arriving from the open sea at the entrance of the canal. With respect to the above, in the present paper, another formulation is considered, in which Miles \& Salmon's (1985) kinematic assumption of a "columnar motion" of the fluid is employed. In accordance with this 
assumption, for the conservative system considered, the governing equations of the problem are derived by means of the Hamilton principle expressed in terms of a material system of coordinates. These coordinates are natural in the variational approach and are especially convenient in the derivation of momentum equations and the formulation of boundary conditions for the model description considered. At the same time, the variational method is of primary importance in an approximate integration of momentum equations by the finite element (FEM) or the finite difference (FDM) methods. With respect to the material coordinates, the free surface is defined by positions of material particles forming this surface within the entire range of time. In other words, the material coordinates of the free surface remain unchanged during the fluid motion. With these coordinates, the boundary conditions at the free surface reduce to a single condition that the pressure at this boundary should be constant (or equal to zero). Thus, the material system of coordinates is found to be superior in our investigations on the initial value problem of long water waves propagating in a canal of variable section.

\section{Formulation of the Problem}

Let us consider a fluid domain shown schematically in Fig. 1. The fluid motion is induced by a piston-type wave maker (rigid wall $B C$ in the figure), which starts to move from rest at a certain moment in time. In order to describe the fluid motion, the Cartesian system of coordinates $(x, y, z)$ is introduced, which denotes the positions of fluid particles in the actual configuration. In the reference configuration, an additional Cartesian system of coordinates is employed in which $(X, Y, Z)$ denote the names of fluid particles. The fluid motion is described by mapping the names into the actual positions of the material points. In order to identify field quantities relative to these independent variables, lowercase letters are used to denote quantities that are expressed in terms of the spatial variables, and uppercase symbols are employed for the material description. Following this notation, for incompressible fluid, the Jacobian of the transformation of the names into their actual positions reads

$$
J=\operatorname{det} \frac{\partial(x, y, z)}{\partial(X, Y, Z)}=1
$$

The fluid domain shown in Fig. 1 is symmetrical with respect to the vertical plane $(A B C D$ in this figure), and therefore in our further discussion only a half of this domain will be taken into account. In general, the canal width $b(X)$ is a continuous function of the $X$ coordinate. In our further discussion, however, this function will be approximated by a set of straight segments, as shown schematically in Fig. 2. With respect to the above, for the long waves considered, a fundamental kinematical assumption is 


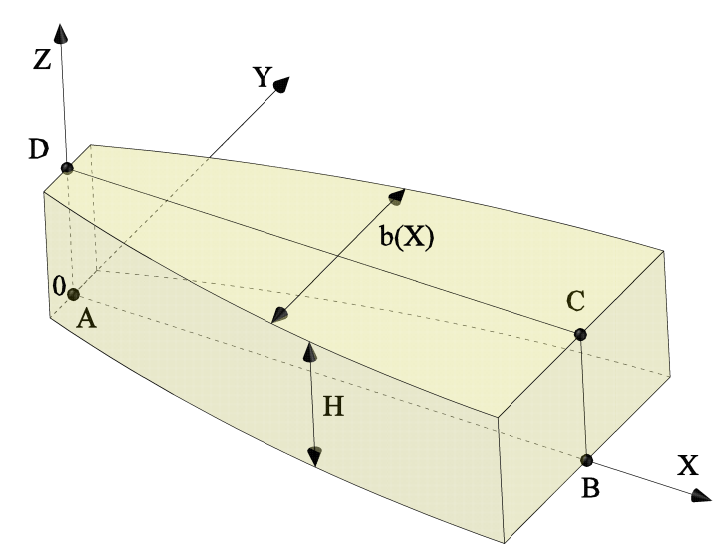

Fig. 1. Fluid domain

employed that the fluid flow may be accurately described by the following displacement field:

$$
\begin{gathered}
x=X+u(X, t), \\
y=Y+v(X, Y, t), \\
z=Z+w(X, Z, t),
\end{gathered}
$$

where $u, v$ and $w$ are components of the displacement vector.

From the first of these equations, it follows that all particles of a given canal section have the same displacements along the canal length. At the same time, because of the variable canal width, these particles undergo displacements perpendicular to the longitudinal canal axis, which are assumed in the following form:

$$
v(X, Y, t)=\frac{1}{b}\left(\frac{\partial b}{\partial X} u+\frac{1}{2} \frac{\partial^{2} b}{\partial X^{2}} u^{2}\right) Y
$$

Finally, the vertical displacements of fluid particles are assumed to be

$$
w(X, Z, t)=\frac{w^{*}(X, t)}{H} Z
$$

where the function $w^{*}(X, t)$ describes the vertical displacements of fluid particles forming the free surface of the fluid.

Substituting these descriptions into equation (1) and performing simple manipulations gives

$$
J=\left(1+u_{, X}\right) \cdot\left(1+v_{, Y}\right) \cdot\left(1+w_{, Z}\right)=f(X, t) \cdot\left(1+\frac{w^{*}}{H}\right)=1 .
$$


Hereinafter, the subscript symbols denote partial derivatives with respect to the independent variables, for instance $u_{, X}=\partial u / \partial X$. From this relation, it follows that

$$
w^{*}=H\left(\frac{1}{f}-1\right) \quad \text { and } \quad w(X, Y, Z, t)=\frac{1-f}{f} Z, \quad 0 \leq Z \leq H .
$$

where

$$
f=1+u_{, X}+v_{, Y}+u_{, X} v_{, Y}
$$

Knowing the displacement field, one may calculate the kinetic energy of the fluid

$$
E_{\text {kin. }}=\frac{1}{2} \rho \int_{X} \int_{Y} \int_{Z}\left[(\dot{u})^{2}+(\dot{v})^{2}+(\dot{w})^{2}\right] J d X d Y d Z,
$$

where $\rho$ is the fluid density, and the dots denote partial differentiation with respect to time. The components of the velocity field in this equation are

$$
\dot{u}=\frac{\partial u}{\partial t}, \quad \dot{v}=\frac{1}{b} \dot{u}\left(b_{, X}+b_{, X X} u\right) Y, \quad \dot{w}=-\frac{\dot{f}}{f^{2}} Z,
$$

where

$$
\dot{f}=\dot{u}_{, X}+\dot{v}_{, Y}+\dot{u}_{, X} v_{, Y}+u_{, X} \dot{v}_{, Y}
$$

From substitution of these relations into equation (8) and integration of the result over the section domain $(Z, Y)=(0-H) \times(0-b)$, the following is obtained:

$$
E_{k i n .}=\frac{1}{2} \rho H \int_{X} b(X)\left[(\dot{u})^{2}+\frac{1}{3}(\dot{u})^{2}\left(b_{, X}+b_{, X X} u\right)^{2}+\frac{1}{3} H^{2}\left(\frac{\dot{f}}{f^{2}}\right)^{2}\right] d X .
$$

The potential energy of the incompressible fluid reads

$$
E_{\text {pot. }}=\rho g \int_{X} \int_{Y} \int_{Z}(z-Z) J d X d Y d Z .
$$

Substitution of (2) and (6) into this equation and integration in the $(Y, Z)$ domain gives

$$
E_{\text {pot. }}=\frac{1}{2} \rho g H^{2} \int_{X} b(X)\left(\frac{1}{f}-1\right) d X .
$$

For the conservative system considered, the Lagrangian density function is equal to the difference between the kinetic and potential energies

$$
\begin{aligned}
L^{*}=E_{\text {kin. }}-E_{\text {pot. }} & =\frac{1}{2} \rho H \int_{X} b(X)\left\{(\dot{u})^{2}\left[1+\frac{1}{3}\left(b_{, X}+b_{, X X} U\right)^{2}\right]+\right. \\
& \left.+\frac{1}{3} H^{2}\left(\frac{\dot{f}}{f^{2}}\right)^{2}+g h\left(1-\frac{1}{f}\right)\right\} d X .
\end{aligned}
$$


As already indicated, the flow description is based on approximations that are admissible for the particular problem considered. Accordingly, in deriving equations of the fluid motion, some simplifications are introduced into the description of this Lagrangian density function. In general, only the terms up to the second order power are taken into account in the integrand. For long waves with small displacements, the derivatives $b_{, X}$ and $b_{, X X}$ are also small quantities, and thus it is justified to employ the following approximations:

$$
\begin{gathered}
\left(b_{, X}+b_{, X X} u\right)^{2} \cong\left(b_{, X}\right)^{2}, \\
\frac{(\dot{f})^{2}}{f^{4}} \cong\left(\dot{u}_{, X}\right)^{2}+\left(\frac{b_{X}}{b}\right)^{2}(\dot{u})^{2}+2 \dot{u}_{, X} \frac{b_{, X}}{b} \dot{u}=\left(\dot{u}_{, X}+\frac{b_{X}}{b} \dot{u}\right)^{2}, \\
\left(1-\frac{1}{f}\right) \cong\left(u_{, X}+\frac{b_{X}}{b} u\right)\left(1-u_{, X}\right)+u^{2}\left[\frac{b_{, X X}}{2 b}-\left(\frac{b_{X}}{b}\right)^{2}\right] .
\end{gathered}
$$

With these approximations, one obtains

$$
\begin{gathered}
L^{*}=E_{\text {kin. }}-E_{\text {pot. }}=\frac{1}{2} \rho H \int_{X}\left[\alpha_{1}(\dot{u})^{2}+\alpha_{2}\left(\dot{u}_{, X}\right)^{2}+\alpha_{3} \dot{u} \dot{u}_{, X}+\right. \\
\left.+\beta_{1} u\left(1-u_{, X}\right)+\beta_{2} u_{, X}\left(1-u_{, X}\right)+\beta_{3} u^{2}\right] d X,
\end{gathered}
$$

where

$$
\begin{gathered}
\alpha_{1}(X)=\left[1+\frac{1}{3}\left(b_{, X}\right)^{2}+\frac{1}{3} H^{2}\left(\frac{b_{, X}}{b}\right)^{2}\right] b, \\
\alpha_{2}(X)=\frac{1}{3} H^{2} b, \quad \alpha_{3}(X)=\frac{2}{3} H^{2} b_{, X}
\end{gathered}
$$

and

$$
\beta_{1}(X)=g H b_{, X}, \quad \beta_{2}(X)=g H b, \quad \beta_{3}(X)=g H\left[\frac{b_{, X X}}{2 b}-\left(\frac{b_{, X}}{b}\right)^{2}\right] b .
$$

In order to obtain the corresponding momentum equation and natural boundary conditions, the Hamilton principle is applied that the action integral should be stationary, i.e. its variation should be equal to zero:

$$
\delta I=\delta \int_{t_{1}}^{t_{2}} L^{*} d t=0
$$


In this formula, the displacement variations at the end time points are equal to zero, i.e. $\left.\delta u\right|_{t_{1}}=\left.\delta u\right|_{t_{2}}=0$. From substitution of equation (16) into this formula, it follows that

$$
\begin{gathered}
\delta I=\frac{1}{2} \rho H \int_{0}^{L}\left\{2 \alpha_{1} \dot{u} \delta \dot{u}+2 \alpha_{2} \dot{u}_{, X} \delta \dot{u}_{, X}+\alpha_{3}\left(\dot{u} \delta \dot{u}_{, X}+\dot{u}_{, X} \delta \dot{u}\right)+\right. \\
\left.+\beta_{1}\left[\left(1-u_{, X}\right) \delta u-u \delta u_{, X}\right]+\beta_{2}\left[\left(1-u_{, X}\right) \delta u_{, X}-u_{, X} \delta u_{, X}\right]+2 \beta_{3} u \delta u\right\} d X=0 .
\end{gathered}
$$

For the linear operations considered, the terms in this integral may be expressed in another form. For example:

$$
\begin{gathered}
\alpha_{1} \dot{u} \delta \dot{u}=\frac{\partial}{\partial t}\left(\alpha_{1} \dot{u} \delta u\right)-\frac{\partial}{\partial t}\left(\alpha_{1} \dot{u}\right) \delta u, \\
\alpha_{2} \dot{u}, X_{, X} \delta \dot{u}_{, X}=\frac{\partial^{2}}{\partial X \partial t}\left(\alpha_{2} \dot{u}_{, X} \delta u\right)-\frac{\partial}{\partial X}\left(\alpha_{2} \ddot{u}_{, X} \delta u\right)-\frac{\partial}{\partial t}\left[\frac{\partial}{\partial X}\left(\alpha_{2} \dot{u}_{, X}\right) \delta u\right]+ \\
+\frac{\partial}{\partial X}\left(\alpha_{2} \ddot{u}_{, X}\right) \delta u .
\end{gathered}
$$

Similar relations hold for the remaining terms in equation (20). Simple manipulations of the variation procedure lead to natural boundary conditions and the following momentum equation:

$$
\left(\alpha_{1}-\frac{1}{2} \frac{\partial \alpha_{3}}{\partial X}\right) \ddot{u}-\frac{\partial}{\partial X}\left(\alpha_{2} \ddot{u}_{, X}\right)-\left(\frac{1}{2} \frac{\partial \beta_{1}}{\partial X}+\beta_{3}\right) u-\frac{\partial}{\partial X}\left(\beta_{2} u_{, X}\right)=0 .
$$

For the case of constant width $b(X)=$ const. of the fluid canal, the momentum equation reduces to the following one:

$$
\ddot{u}-\frac{1}{3} H^{2} \ddot{u}_{, X X}-g H u_{, X X}=0 .
$$

Equation (22) is a linear partial differential equation with functional coefficients. In order to integrate this equation in the time-space domain, it is reasonable to resort to a numerical integration in which continuous variables are replaced by a set of discrete values of them at discrete points in time and space. In this discrete integration, for instance by means of the finite difference method (FDM), the space distribution of a function is replaced by its point values at equally spaced nodal points, and the space derivatives are replaced by difference quotients. It should be stressed, however, that such a procedure is not unique. A better way is to return to the action integral and approximate the continuous Lagrangian density function by a set of trial functions dependent on the nodal values of dependent variables. Such a procedure enables us to calculate the space integral and express this Lagrangian function in terms of dependent variables at discrete points in space. In this case, the variation procedure is applied to a set of these variables directly. 


\section{Discrete Formulation and Direct Variational Approach}

With respect to the notations shown in Fig. 2, let us consider the equal spacing of nodal points along the fluid length $L=N \cdot a$ and a typical nodal point $k$ with $X_{k}=k \cdot a$. Together with this point, its neighbor points $k-1$ and $k+1$ are taken into account. The Lagrangian density function is calculated as the sum of integrals calculated over subdomains defined by the assumed grid. Thus, for our needs, it is sufficient to consider only one segment $(k-1)-k$ and calculate the integral as dependent on the unknown nodal values of the dependent variable. In order to simplify the integration, the fluid width is approximated by a set of straight segments, and the distribution of variables over one segment is described by linear functions. With these approximations, the following relations hold:

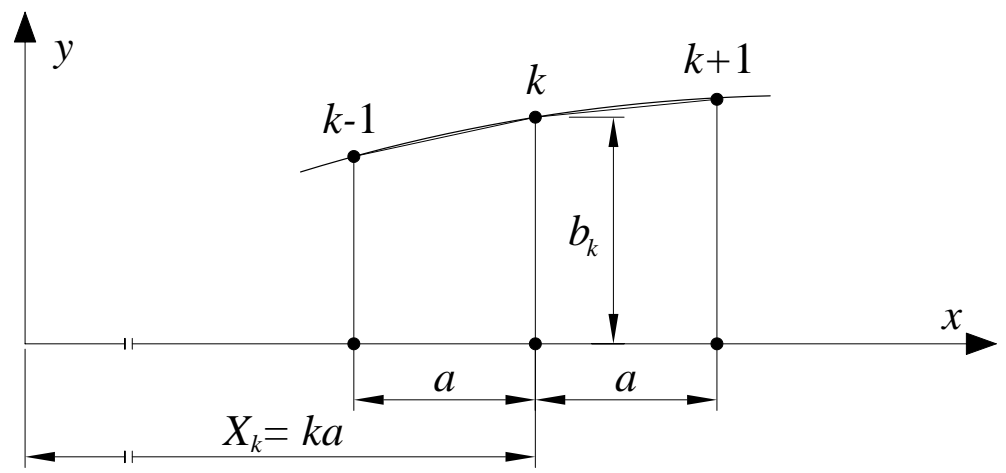

Fig. 2. Spacing of points in a canal of variable section

$$
\begin{gathered}
b_{, X}=\frac{1}{a}\left(b_{k}-b_{k-1}\right), \quad b_{, X X}=0 \\
\frac{b_{, X}}{b} \cong \frac{2}{a} \frac{b_{k}-b_{k-1}}{b_{k}+b_{k-1}}, \quad k=1,2, \ldots, N
\end{gathered}
$$

For an individual segment, equation (16) gives

$$
K=a \int_{0}^{1}\left[\alpha_{1}(\dot{u})^{2}+\alpha_{2}\left(\dot{u}_{, X}\right)^{2}+\alpha_{3} \dot{u}_{, X}-\beta_{1} u u_{, X}-\beta_{2}\left(u_{, X}\right)^{2}-\beta_{3} u^{2}\right] d \xi,
$$

where the local coordinate $X=a \cdot \xi$ has been employed.

The parameters $\alpha_{1}, \cdots, \beta_{3}$ in this equation are expressed by

$$
\begin{aligned}
& \alpha_{1}(\xi)=\alpha_{1}^{*} b(\xi), \quad \alpha_{2}(\xi)=a_{2}^{*}(\xi) b(\xi), \quad \alpha_{3}=\text { const }, \\
& \beta_{1}=\text { const }, \quad \beta_{2}=\beta_{2}^{*} b(\xi), \quad \beta_{3}=\beta_{3}^{*} b(\xi) .
\end{aligned}
$$


In order to calculate the value of integral (25), it is sufficient to consider only the first three terms in it, for which we have

$$
\begin{gathered}
K 1=a \alpha_{1}^{*} \int_{0}^{1} b(\xi)[\dot{u}(\xi)]^{2} d \xi= \\
=\frac{a \alpha_{1}}{12}\left[\left(3 b_{k-1}+b_{k}\right)\left(\dot{u}_{k-1}\right)^{2}+2\left(b_{k-1}+b_{k}\right) \dot{u}_{k-1} \dot{u}_{k}+\left(b_{k-1}+3 b_{k}\right)\left(\dot{u}_{k}\right)^{2}\right], \\
K 2=a \alpha_{2}^{*}\left(\frac{\dot{u}_{k}-\dot{u}_{k-1}}{a}\right)^{2} \int_{0}^{1} b(\xi) d \xi=\frac{\alpha_{2}^{*}}{2 a}\left(\dot{u}_{k}-\dot{u}_{k-1}\right)^{2}\left(b_{k-1}+b_{k}\right), \\
K 3=a \alpha_{3}\left(\frac{\dot{u}_{k}-\dot{u}_{k-1}}{a}\right) \int_{0}^{1} \dot{u}(\xi) d \xi=\frac{\alpha_{3}}{2}\left[\left(\dot{u}_{k}\right)^{2}-\left(\dot{u}_{k-1}\right)^{2}\right] .
\end{gathered}
$$

For the remaining terms in equation (25), the same formulae hold except for the dots, which should be canceled out, and the constants $\alpha$ should be replaced by the constants $\beta$. The discrete procedure enables us to express equation (16) in the form of a sum of integrals (25) calculated for individual segments. The final result of these integrations is a linear combination of nodal displacements and velocities, which are dependent parameters of the problem. Now, the standard variation procedure is applied to these nodal parameters. For instance, in place of the first of equations (19), we have

$$
\dot{u} \delta \dot{u} \rightarrow \dot{u}_{k} \delta \dot{u}_{k}=\frac{\partial}{\partial t}\left(\dot{u}_{k} \delta u_{k}\right)-\ddot{u}_{k} \delta u_{k} .
$$

The final result of the variation procedure is a set of equations corresponding to the set of unknown nodal displacements. A typical equation for the $k$ node reads

$$
A_{1} \ddot{u}_{k-1}+A_{2} \ddot{u}_{k}+A_{3} \ddot{u}_{k+1}+B_{1} u_{k-1}+B_{2} u_{k}+B_{3} u_{k+1}=0 \text {, }
$$

where:

$$
\begin{aligned}
& A_{1}=\frac{1}{6}\left(b_{k-1}+b_{k}\right) \cdot(\left.\frac{6}{a} \alpha_{2 L}^{*}+a \alpha_{1 L}^{*}\right), \quad A_{3}=\frac{1}{6}\left(b_{k}+b_{k+1}\right) \cdot\left(\frac{6}{a} \alpha_{2 R}^{*}+a \alpha_{1 R}^{*}\right), \\
& A_{2}=-\frac{a}{6} \alpha_{1 L}^{*}\left(b_{k-1}+3 b_{k}\right)-\frac{a}{6} \alpha_{1 R}^{*}\left(3 b_{k}+b_{k+1}\right)-\frac{\alpha_{2 L}^{*}}{a}\left(b_{k-1}+b_{k}\right)- \\
& \quad-\frac{\alpha_{2 R}^{*}}{a}\left(b_{k}+b_{k+1}\right)-\left(\alpha_{3 L}-\alpha_{3 R}\right), \\
& B_{1}=\frac{1}{6}\left(b_{k-1}+3 b_{k}\right) \cdot\left(\frac{6}{a} \beta_{2 L}^{*}+a \beta_{3 L}\right), \quad B_{3}=\frac{1}{6}\left(b_{k}+b_{k+1}\right) \cdot\left(\frac{6}{a} \beta_{2 R}^{*}+a \beta_{3 R}\right), \\
& B_{2}=\frac{a}{6} \beta_{3 L}\left(b_{k-1}+\right.\left.3 b_{k}\right)+\frac{a}{6} \beta_{3 R} \alpha_{1 R}^{*}\left(3 b_{k}+b_{k+1}\right)-\frac{\beta_{2 L}^{*}}{a}\left(b_{k-1}+b_{k}\right)- \\
&-\frac{\beta_{2 R}^{*}}{a}\left(b_{k}+b_{k+1}\right)-\left(\beta_{1 L}-\beta_{1 R}\right),
\end{aligned}
$$


In these relations, the subscript $L$ (left) denotes values for the segment $(k-1)-$ $k$, and the symbol $R$ (right) the corresponding values for the segment $k-(k+1)$. Equations (29) are written for all nodal points: $X_{k}=k \cdot a, k=1,2, \ldots, N, L=(N+$ $1) \cdot a$. The final result is written in the form of the matrix equation

$$
\boldsymbol{A M} \cdot \ddot{\boldsymbol{U}}+\boldsymbol{B} \boldsymbol{M} \cdot \boldsymbol{U}=\boldsymbol{P},
$$

where $\boldsymbol{A} \boldsymbol{M}$ and $\boldsymbol{B} \boldsymbol{M}$ are square matrices, and $\boldsymbol{P}$ is a vector matrix dependent on the generator motion. This equation is integrated in time by means of a discrete Wilson $\theta$ method (Bathe, 1982). With this method, instead of the continuous time, a sequence of time steps with the increment $\Delta t>0$ is considered. A solution at the next step in time depends on a known solution at the previous step in time.

\section{Numerical Simulations}

The approximate solution presented in the previous sections is applied to particular cases of the generation of water waves in a canal of variable section. Integration of the momentum equation enables us to calculate the horizontal displacement of the fluid sections and thus the free surface evolution in time for a specified generator displacement $x g(t)$. With respect to periodic motions observed under natural conditions, the harmonic generation of waves is of primary importance. Since we are dealing with motion starting from rest, harmonic generation is obtained as a limiting case of generation described by the formula (Wilde and Wilde, 2001)

$$
x g(t)=A m\left[A_{3}(\tau) \cos \omega t+D_{3}(\tau) \sin \omega t\right],
$$

where

$$
\begin{gathered}
A_{3}(\tau)=\frac{\tau^{3}}{3 !} \exp (-\tau) \\
D_{3}(\tau)=1-\left(1+\tau+\frac{\tau^{2}}{2 !}+\frac{\tau^{3}}{3 !}\right) \exp (-\tau), \quad \tau=\eta t
\end{gathered}
$$

In these equations, $\tau$ is a non-dimensional time, $t$ means time, and $\eta$ is a memory parameter responsible for a growth in time of the generator displacement. One can check that, at the starting point $t=0$, the displacement velocity and acceleration of the generator plate are all equal to zero. Moreover, with increasing time, the generator goes asymptotically to harmonic motion with constant amplitude ( $A m$ in equation 32 ).

In the first step, in order to assess the overall accuracy of the formulation, numerical calculations are made for cases corresponding directly to hydraulic experiments carried out in a laboratory flume. The laboratory flume is shown schematically in Fig. 3.

Water waves are generated by a piston-type generator placed at the right hand side of the flume, which starts to move at a certain point in time. For the finite fluid domain and a limited elapse of time measured from this starting point, the solution in this 
domain mimics a solution in an infinite fluid area. In this case, we have the unsteady problem of purely propagating water waves. On the other hand, after a sufficiently long elapse of time measured from this starting point, a generated wave arrives at the left boundary, and then a reflected wave is created. In the case of a semi-infinite fluid domain, a reflection of steady harmonic waves arriving at this boundary leads to the steady problem of standing water waves. In laboratory experiments, we usually deal with a flume of finite length, and thus, for a long elapse of time from the starting point, the picture of the free surface represents a more complicated unsteady surface evolving in time.

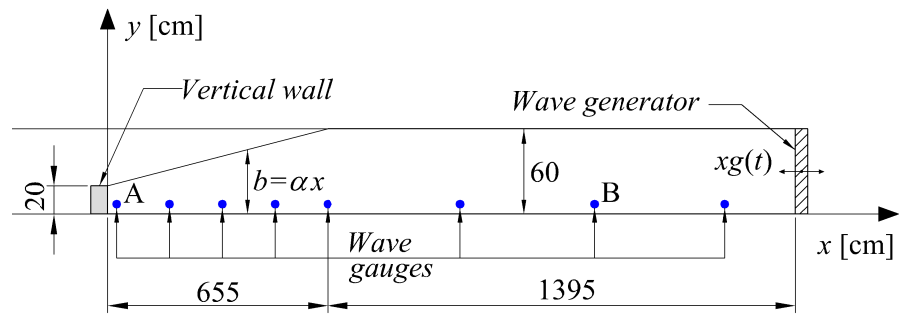

Fig. 3. A scheme of a laboratory flume

Numerical solutions and laboratory experiments were performed for a selected set of generations corresponding to the assumed lengths and heights of surface waves. Some of the results obtained in numerical simulations and laboratory experiments are illustrated in Fig. 4, where the plots represent the free-surface elevation at the left boundary. From the plots, it may be seen that the theoretical solution describes the overall features of the phenomenon fairly well. At the same time, however, the results of this theoretical model exceed those of laboratory experiments. This discrepancy results from the approximate description of the phenomenon, which follows the fundamental assumption of a conservative system and a "columnar" fluid motion. Such approximations, however, are justified in practical applications.

The solutions developed above are employed in our further investigations on the problem of the enforcement of wave height by means of a structure installed in an offshore zone. For practical reasons, it would be desirable to define the effectiveness of such a structure. A good measure of this effectiveness is the ratio of the accessible water energy of a water column of unit section near the structure to the energy of this column without this structure. Since the available water energy depends mainly on the heights of water waves, the free-surface elevation is taken as the basic parameter of the effectiveness.

A remark is needed. Waves arriving at beach zones from the open sea undergo transformations associated with gradual changes in water depth. Their elevation at a particular point in the coastal zone is regarded as a free elevation (without a structure). 


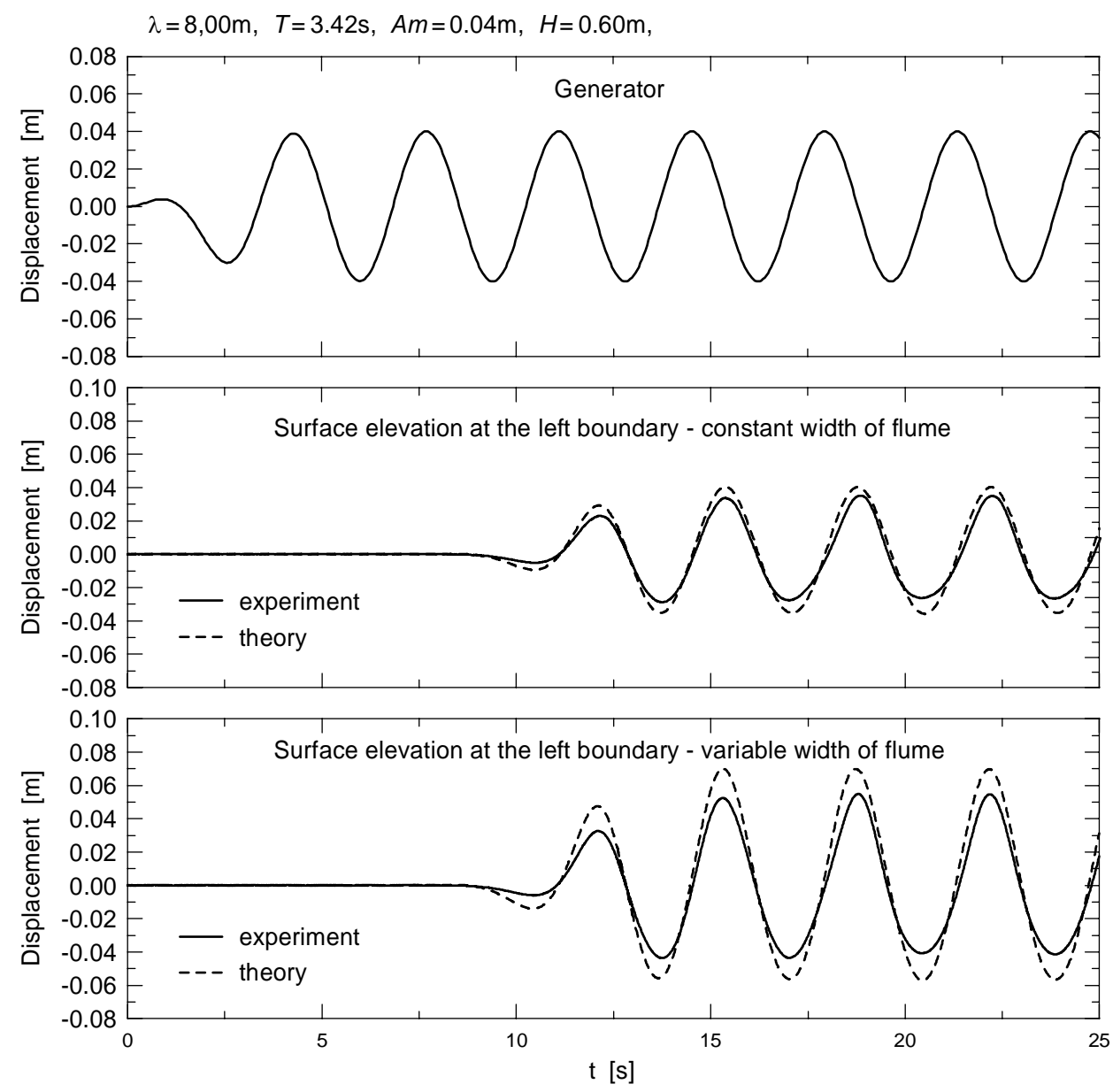

Fig. 4. Comparison of the theoretical solution with data obtained in laboratory experiments

In the case of a rigid vertical breakwater (vertical wall) placed in this zone, the arriving waves reflect from this structure, and thus we have standing waves with their own height greater than the height of propagating waves. If $h_{F}$ (point B, Fig. 3) denotes the water wave height in the fluid domain free from a structure, $h_{C}$ (point A, Fig. 3) is the height of water waves close to a structure for a canal of constant section, and $h_{S}$ (point A, Fig. 3) is this height for a canal of variable ("triangular") section, then the following set of ratios is taken into account:

$$
\mu_{1}=\frac{h_{C}}{h_{F}}, \quad \mu_{2}=\frac{h_{S}}{h_{F}}, \quad \mu_{3}=\frac{\mu_{2}}{\mu_{1}} .
$$

For the energy problem considered, the effectiveness ratio of a structure is defined as the square of the ratios defined above, i.e. $E_{R}=\left(\mu_{i}\right)^{2}, i=1,2,3$. 
Table 1. Relative height of waves measured at points $A$ and $B$ of the canal (see Fig. 3)

\begin{tabular}{|c|c|c|c|c|c|c|}
\hline$\lambda$ & \multicolumn{3}{|c|}{ Theory } & \multicolumn{3}{c|}{ Experiment } \\
\cline { 2 - 7 }$[\mathrm{m}]$ & $\mu_{1}$ & $\mu_{2}$ & $\mu_{3}$ & $\mu_{1}$ & $\mu_{2}$ & $\mu_{3}$ \\
\hline 1 & 2 & 3 & 4 & 5 & 6 & 7 \\
\hline 4.00 & 1.901 & 3.513 & 1.848 & 1.978 & 3.082 & 1.558 \\
\hline 5.00 & 2.033 & 3.500 & 1.722 & 2.053 & 2.991 & 1.457 \\
\hline 6.00 & 2.040 & 3.420 & 1.676 & 1.954 & 3.053 & 1.562 \\
\hline 7.00 & 2.023 & 3.372 & 1.667 & - & - & - \\
\hline 8.00 & 2.054 & 3.405 & 1.658 & 1.990 & 3.046 & 1.530 \\
\hline 10.00 & - & - & - & 1.972 & 2.996 & 1.519 \\
\hline
\end{tabular}

With respect to laboratory experiments carried out in a hydraulic flume, the structure is constructed in the form of a rigid, vertical wall forming the left boundary of the flume. Two cases are considered. The first corresponds to the variable canal shown in Fig. 3, and in the second case, the canal has constant section. The laboratory experiments and the associated numerical solutions were performed for selected frequencies of the generator motion (selected lengths of the generated water waves). The results of the numerical calculations and laboratory experiments were used in calculating the ratios of water heights as defined by the above formulae. The effectiveness ratios for particular cases considered are shown in Table 1.

From the data collected in this table, it follows that a structure in the form of a convergent canal can enforce the height of a surface wave reflected from this structure. The structure effectiveness strongly depends on the length of the wave arriving at and reflected from this structure. The data in this table may be used in assessing the accuracy of the theoretical solution by comparing its results with those obtained in the hydraulic experiments.

In order to obtain a better insight into the formulation developed above and to estimate the effectiveness of structures, additional examples are presented in the subsequent figures (5) and (6). The plots in Fig. 5 show the evolution in time of the free surface at the left boundary of the fluid domain for canals of variable and constant sections. The consecutive plots correspond to selected lengths of the generated waves. From the plots, it may be seen that the wave heights in the canal of variable section exceed those in the canal of constant section by about $50 \%$. The ratio of the wave height for the canal of variable section to the wave height for the canal of constant section depends on the wave length. A similar comparison is presented in Fig. 6, where the plots show the evolution in time of the free surface at the left boundary and at a point $15 \mathrm{~m}$ away from this boundary ( $5 \mathrm{~m}$ from the generator plate). For a finite elapse of time measured from the starting point, the free-surface elevation at this point ( $5 \mathrm{~m}$ from the generator plate) corresponds directly to a harmonic wave propagating freely in a fluid of constant depth. From the plots in Fig. 5 and 6, it may be seen that the effectiveness ratio is smaller for longer waves. The laboratory and numerical experiments show that a simple structure placed in the coastal zone may improve the utilization of energy carried by water waves. 

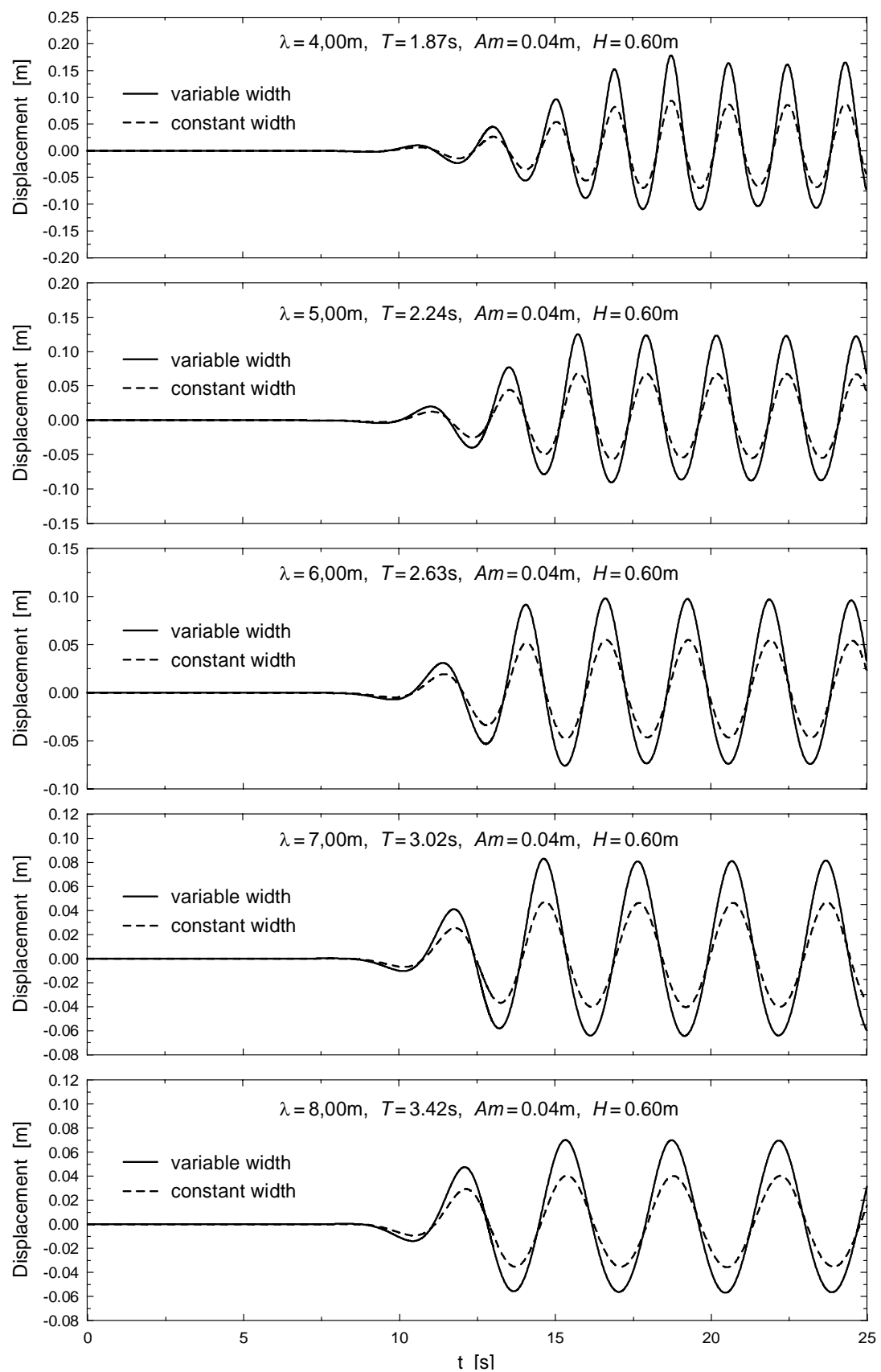

Fig. 5. Evolution in time of the free-surface elevation at the left boundary 

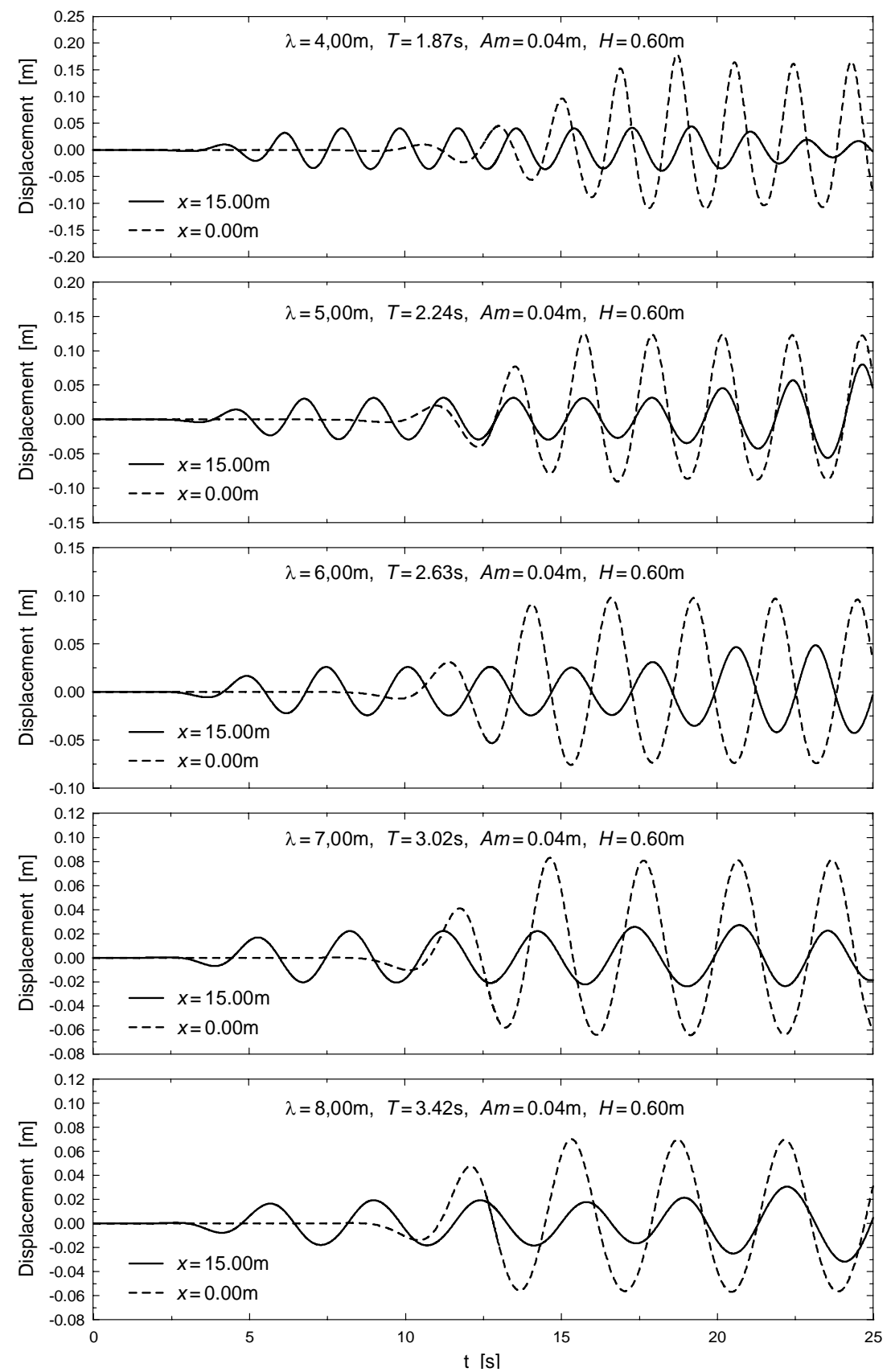

Fig. 6. Comparison of the free-surface elevations at two sections of the flume 


\section{Concluding Remarks}

The theoretical description of long water waves propagating in a canal of variable section was formulated with respect to material variables, which simplify the variational analysis and the solution of boundary conditions, especially at the free surface of the fluid. The formulation developed in this paper makes it possible to reduce the description of a three-dimensional physical problem to a one-dimensional problem in space. Obviously, such a reduction is only an approximation in the description of the original task. In order to assess the accuracy of the formulation, its results were compared with data obtained in experiments performed in a laboratory flume. From the comparison, it follows that, for the long waves considered, the simplified model gives sufficiently accurate results. With respect to this, the model description was applied to the solutions of particular cases of wave generation dependent on the height and length of water waves. The results of numerical calculations show that a simple structure in the form of a convergent canal installed in an offshore sea zone can enforce the wave height of a standing wave occurring due to this structure. It is important to note that, with such a structure, the theoretical power of sea waves that can be utilized exceeds several times the power of sea waves propagating freely within shallow waters.

\section{References}

Bathe K. J. (1982) Finite Element Procedures in Engineering Analysis, Prentice Hall Inc. Englewood Cliffs, New Jersey.

Lamb H. (1975) - Hydrodynamics, Cambridge University Press.

McLachlan N. W. (1964) Bessel functions for engineers - Polish Edition, PWN, Warszawa.

Miles J. and Salmon R. (1985) Weakly dispersive nonlinear gravity waves, J. Fluid Mechanics, vol. 157, 519-531.

Wilde P. and Wilde M. (2001) On the generation of water waves in a flume, Archives of Hydro-Engineering and Environmental Mechanics, 48 (4), 69-83. 